\title{
EDUCACIÓN Y EMPLEO: LA SITUACIÓN HISTÓRICA DE LOS J ÓVENES EN ARGENTINA, 1970-2001
}

\author{
Ana Miranda, Analía Otero y Agustina Corica \\ Facultad Latinoamericana de Ciencias Sociales - Sede Argentina \\ amiranda@flacso.org.ar
}

\section{Resumen}

El objetivo del presente trabajo es realizar un análisis de la información demográfica y de los principales indicadores laborales y educativos de la población comprendida entre los jóvenes de 15 a 29 años. La información en análisis se corresponde con los Censos Nacionales de Población y Vivienda de los años 1970, 1980, 1991 y 2001 para el total del país. A partir de dicha información, se realizó una comparación histórica y sistemática de los indicadores laborales y educativos por grupo etario, dando cuenta de las principales tendencias verificadas en ambos. Este análisis nos permitió brindar un panorama histórico de los cambios que se fueron sucediendo en la situación de los jóvenes en la Argentina a lo largo de los últimos 30 años.

\section{Introducción}

Los cambios políticos, económicos y sociales registrados a lo largo de las últimas tres décadas aventuran modificaciones sustantivas respecto a los modos y las condiciones de vida de los jóvenes. En correspondencia, en el campo de la sociología de la juventud se identifica un incremento de los aportes que desde diferentes perspectivas teóricas y metodológicas, abordan cómo han impactado dichas transformaciones en el sector juvenil.

Dentro del campo de la sociología de la juventud, las investigaciones que han estudiado las transiciones de los jóvenes hacia la vida adulta apuntan que hasta mediados del siglo XX la principal actividad de los y las jóvenes estaba asociada a la integración al mercado laboral. En este sentido, se ha observado que hasta los años setenta los recorridos de los jóvenes se habían estructurado en torno a trayectos estandarizados: el paso de la educación al empleo entre los hombres, y el paso de la educación al cuidado de los hijos y el hogar entre las mujeres. Dichos recorridos, estaban mediatizados por las posiciones diferenciales de los jóvenes en la estructura social. No obstante, las transformaciones de los últimos años modificaron ampliamente los recorridos juveniles, lo cual ha estimulado nuevas reflexiones sobre los procesos de transición hacia la vida adulta.

Sobre todo, a partir de la década del noventa, y en directa relación con la emergencia de nuevas problemáticas sociales -tales como la crisis del empleo protegido y a tiempo indeterminado- las interpretaciones teóricas advirtieron una desestandarización de los itinerarios y trayectorias juveniles. Respecto a estas últimas, se ha señalado que lejos de continuar correspondiéndose con los trayectos estandarizados a modo de antaño, las transiciones de los jóvenes han tendido a prolongarse y diversificarse. Por ejemplo, el tránsito entre la educación y el empleo parece haberse complejizado de tal forma que para muchos jóvenes supone más que un momento, un proceso de larga duración. Ese proceso puede comprender pasajes por una diversidad de situaciones, como trayectos en trabajos de corta duración, períodos de desempleo, períodos de inactividad, etc.

En la sociedad argentina, desde mediados de los setenta presenciamos un escenario de significativos cambios, entre ellos, las transformaciones operadas en el perfil productivo y en el mercado laboral. En paralelo a un contexto de agudo deterioro laboral, se evidenció una tendencia hacia la mayor escolarización de la población. Ambos procesos suponen modificaciones en la situación de la población y de los jóvenes en particular. En diversas investigaciones se enfatizó que los jóvenes fueron uno de los grupos más afectados por las reestructuraciones de la estructura ocupacional. Se advirtió, además, que la distribución de esos impactos no es homogénea sino que fueron aquellos jóvenes que provienen de sectores de menor capital educativo y menores ingresos quienes sufrieron las consecuencias más severas. Al mismo tiempo, puede advertirse que estos impactos son diferentes si consideramos distintos grupos de edad al interior del conjunto de la categoría joven.

A lo largo del presente texto, trabajaremos sobre la base de datos del Censo Nacional de Población y Viviendas registrados por el Instituto Nacional de Estadística y Censos (INDEC). Y, a los efectos del análisis estadístico, utilizaremos el criterio europeo que considera a la juventud como la población comprendida entre 15 y 29 años de edad. Frente a la amplitud de edades y a la diversidad de etapas que abarca dicha categoría, distinguimos en tres subgrupos etarios: el primero abarca los jóvenes de 15 a 19 años (jóvenes menores), el segundo comprende a aquellos de 20 a 24 años (jóvenes plenos) y el tercero a jóvenes de 25 a 29 años de edad (jóvenes adultos).

El artículo se organiza en cuatro apartados, en un primer apartado haremos una breve descripción de la evolución de la población joven en Argentina y su peso específico durante las últimas tres décadas. Luego, avanzamos en la exposición de tendencias respecto a la participación de los jóvenes en el sistema educativo formal. En el apartado siguiente, exponemos datos significativos en torno a su participación en el mercado laboral. Por último, en relación con el análisis realizado elaboramos un conjunto de consideraciones finales.

\section{Evolución de la población de joven en Argentina}


Para caracterizar a la población joven, comenzamos por considerar el peso específico de este grupo poblacional. En valores absolutos en 1970 la población total del país ascendía a 23.390.050 de habitantes, de los cuales 5.751 .900 eran jóvenes de 15 a 29 años de edad. De modo que, para entonces, los jóvenes representaban un $24,5 \%$ de la población. Considerando los distintos subgrupos etarios la población joven se distribuía de la siguiente forma: 2.098 .700 (12,6\%) de jóvenes menores, 1.950 .500 (11,8\%) de jóvenes plenos y 1.702.700 (10,3\%) de jóvenes adultos (ver Anexo, cuadro № 1).

Siguiendo la evolución de dicha población a partir de los relevamientos censales realizados durante las dos décadas siguientes, se puede señalar que en su conjunto la población joven sufrió variaciones de escasa magnitud. Más específicamente, durante los períodos de 1980 y 1991 la población de 15 a 29 años comprendió 6.689.928 y 7.608.470 de habitantes, respectivamente. A la luz de estos datos se advierte que fueron leves las modificaciones en cuanto al peso de este sector en el total de la población. Asimismo, considerando la información desagregada entre los distintos subgrupos etarios de jóvenes es posible corroborar que también fueron menores las diferencias porcentuales registradas durante ambos períodos.

Finalmente a principios del siglo XXI en Argentina, los jóvenes de 15 a 29 años comprendieron 9.082 .984 de habitantes sobre un total de población de 36.260.130. Así, los jóvenes llegaron a representar una proporción del 25\%, en esta sociedad. Traducido al interior de los subgrupos etarios durante el 2001, su modo de distribución ha sido el siguiente: la población de jóvenes menores alcanzó 3.188.304 (12,2\%), los jóvenes plenos 3.199.339 (12,3\%), mientras que para los jóvenes adultos se registró un 2.695 .341 de habitantes $(10,3 \%)$.

Ahora bien, a partir de una mirada de este sector tomando en cuenta la variable género, se advierte que su distribución es homogénea. Y en cuanto a la evolución que se registra a través de los diferentes períodos, las proporciones se mantienen presentando leves variaciones entre la población masculina y femenina. En base al relevamiento censal de la década de 1970, la población de varones de 15 a 29 años de edad era de 2.871 .350 y de mujeres 2.880.550; mientras que durante el 2001 estos valores alcanzaron al 4.540 .462 y 4.542.522 de varones y mujeres, respectivamente.

En síntesis, aunque en términos absolutos entre los relevamientos intercensales se observa un leve incremento poblacional entre los jóvenes, destacamos que a lo largo del período bajo análisis 1970-2001 el peso específico de la población joven se ha mantenido relativamente constante. En este sentido, cabe remarcar que como queda expuesto en el cuadro № 1, desde mitades del siglo XX hasta la actualidad, la población de jóvenes en Argentina representa alrededor del 25\%, es decir, un cuarto de la población total del país.

\section{Cuadro № 1}

Evolución porcentual de la población de jóvenes entre 15 y 29 años - porcentajesTotal del País

\begin{tabular}{|c|c|c|c|c|c|}
\hline & 1970 & 1980 & 1991 & 2001 & $\begin{array}{c}\text { Diferencia \% } \\
\text { entre } 2001- \\
1970\end{array}$ \\
\hline 15 a 19 años & 12.69 & 12.03 & 12.59 & 12.26 & -0.43 \\
\hline 20 a 24 años & 11.80 & 11.43 & 10.84 & 12.30 & 0.50 \\
\hline 25 a 29 años & 10.30 & 10.91 & 10.18 & 10.36 & 0.07 \\
\hline \multicolumn{6}{|c|}{ HOMBRES } \\
\hline 15 a 19 años & 13.00 & 12.40 & 13.01 & 12.95 & -0.05 \\
\hline 20 a 24 años & 11.91 & 11.62 & 11.14 & 12.83 & 0.92 \\
\hline 25 a 29 años & 10.35 & 11.09 & 10.44 & 10.67 & 0.33 \\
\hline \multicolumn{6}{|c|}{ MUJ ERES } \\
\hline 15 a 19 años & 12.39 & 11.68 & 12.19 & 12.55 & 0.16 \\
\hline 20 a 24 años & 11.68 & 11.25 & 10.56 & 12.75 & 1.07 \\
\hline 25 a 29 años & 10.25 & 10.74 & 9.93 & 10.88 & 0.63 \\
\hline
\end{tabular}

Fuente: Elaboración propia en base a datos del Censo Nacional de Población y Viviendas del INDEC

\section{Tendencias educ ativas en la población joven}

En este apartado presentamos las principales tendencias nacionales respecto a la escolarización de la población joven. Trabajamos a partir de los datos de los distintos niveles de enseñanza alcanzados por esta población en el período comprendido entre 1970-2001. La información relevada nos permitió observar a largo plazo, cómo han sido los alcances de esta distribución para los distintos subgrupos de jóvenes.

Antes de adentrarnos en el análisis de los datos, es necesario señalar que la estructura del sistema educativo formal ha sufrido modificaciones sustantivas durante los años '90. Históricamente, la estructura del sistema educativo argentino estuvo organizada en distintos niveles, un primer nivel inicial o primario, que abarcaba 7 años, (con límites etarios teóricos entre los 6 y los 12 años de edad); un segundo nivel secundario de 5 años (entre los 13 y 18 años de edad) y un nivel de educación superior (terciario y 
universitario). Mientras se mantuvo vigente dicha estructura, la obligatoriedad alcanzaba sólo al nivel de educación primaria de enseñanza.

A partir de la aplicación de la Ley Federal de Educación No 24.195 sancionada en 1993, la estructura del sistema educativo fue modificada. Esta Ley definió una nueva estructura organizada en un nivel inicial (1 año), tres ciclos de Educación General Básica (9 años), Polimodal (3 años) y educación superior.

Producto de esta reforma (1), se extendió la obligatoriedad, quedando establecida en 10 años de escolarización, que comprenden el nivel inicial y la Educación General Básica (EBG).

A lo largo del período, en términos generales, se verifica un incremento en el nivel educativo alcanzado de la población joven. Sin embargo, este incremento se traduce en forma diferencial al interior de los distintos subgrupos de jóvenes y pueden indicarse particularidades para cada uno de ellos.

A partir de la lectura de los datos censales en 1970 el grupo de jóvenes menores -15 a 19 años- habían alcanzado el nivel primario completo en un $87 \%$ y sólo el 5,9\% el nivel medio. Mientras que, durante el 2001 el 88,1\% hacía completado el nivel primario y el $11,8 \%$ el nivel medio. Por ende, y como queda expuesto en el gráfico $N^{0}$ 1, es en la educación media donde se registran las mayores variaciones.

Por otra parte, entre los jóvenes menores puede observarse que en cuanto al comportamiento según género no se registraron notorias diferencias, aunque sí puede verificarse un incremento mayor del nivel educativo alcanzado por las mujeres. En esta dirección, en las mujeres ese incremento fue del $11,5 \%$ y en el caso de los varones sólo del 4,8\% (ver anexo).

\section{Gráfico № 1 \\ J óvenes entre 15 y 19 años según máximo nivel educativo alcanzado Total del País}

Fuente: Elaboración propia en base a datos de los Censos Naciones de Población y Viviendas del INDEC

En igual sentido, entre los jóvenes plenos se puede corroborar que existe tendencialmente un incremento respecto al nivel educativo. Mientras que en 1970 el 74,5\% alcanzaba hasta primaria completa y un 20,3\% el secundario completo; en 2001 un 45,7\% obtenía secundaria completa incrementándose así el porcentaje de jóvenes con título secundario en un 23\%. Al mismo tiempo, se verificó un incremento en el porcentaje de jóvenes con título superior completo, este valor ascendió a un $2 \%$ de la población del subgrupo durante el último período censal. Tomando en cuenta ambas tendencias cabe destacar que es entre los jóvenes plenos donde se registró el mayor incremento respecto a los niveles educativos alcanzados.

Es decir que, frente a los datos del censo podemos observar una expansión proporcional de los certificados del nivel educativo medio entre los jóvenes de 20 a 24 años de edad, sobre todo en el último período. Justamente, los datos de 2001 nos indican que la proporción de jóvenes con certificado de nivel secundario duplica a la de los adultos (ver Gráfico $N^{\circ} 4$ ). Pese a ello, los datos son también elocuentes en registrar que en la misma medición más de 5 de cada 10 jóvenes de ese subgrupo de edad no contaba con un diploma de nivel educativo medio (ver anexo).

Al interior de este subgrupo, si comparamos la distribución según género se advierte que es mayor el porcentaje de mujeres que han completado el nivel medio. En 1970 el 22,3\% de ellas habían alcanzado hasta secundario completo mientras que para el caso de los varones ese porcentaje asumió el 18,3\%; dos décadas después ambos porcentajes ascendían al 49,8\% y 41,5\%, respectivamente. En lo que hace a la evolución del nivel de educación superior, un dato importante a señalar es que entre las mujeres se identifica el mayor incremento (de 1,4\%, 1970 a 4,3\%, 2001).

En 2001, prácticamente el $50 \%$ de las mujeres obtuvo el título secundario y los varones alrededor del $40 \%$, la diferencia porcentual entre ambos géneros es casi de 10 puntos. Cabe señalar que en correspondencia con lo expuesto por otras investigaciones, se corrobora la existencia de una acentuación de la feminización de la matricular escolar (ver Anexo I, gráfico Nº 2). Asimismo, se confirma la tendencia señalada sobre el alto porcentaje de varones y mujeres que no obtuvieron un certificado del nivel medio de enseñanza.

\section{Gráfico № 2 \\ J óvenes entre 20 y 24 años según máximo nivel educativo alcanzado \\ Total del País}

Fuente: Elaboración propia en base a datos de los Censos Naciones de Población y Viviendas del INDEC.

En cuanto al subgrupo de jóvenes adultos -25 a 29 años-, se observa entre 1970-2001 una misma direccionalidad con relación al incremento de los años de escolarización. Para este caso, como queda expuesto en el gráfico № 3, en 1970 el 77,6\% alcanzó primaria completa, el 18,3\% obtuvo un certificado medio de enseñanza y en un $4 \%$ un certificado de nivel superior. A partir de los datos censales del 2001, la información nos muestra un aumento en el porcentaje de los jóvenes que completaron el nivel medio, siendo esta variación del 18,9\%.

Nuevamente, es significativa la porción de jóvenes que no obtuvo el certificado de nivel medio de enseñanza. En relación con esta 
cuestión, podemos decir que el alcance en materia de logros educativos aún presenta dificultades para gran parte de los jóvenes de nuestra sociedad. En este sentido, los datos son elocuentes, 5 de cada 10 jóvenes adultos, al igual que para los jóvenes plenos, no finalizó la escuela secundaria, proporción que de este modo se mantiene constante para ambos subgrupos.

Por otra parte, la diferencia entre géneros respecto de la obtención del diploma secundario es menor que en los otros grupos de edad. Al respecto, el $38 \%$ de las mujeres jóvenes y $36,2 \%$ de los varones obtuvieron el secundario completo. $\mathrm{Y}$, es en el caso de la obtención del título de nivel superior donde se pueden observar las mayores variaciones. De forma tal que, las mujeres duplican proporcionalmente la tasa de egresados en este nivel educativo. El porcentaje alcanzado por las mujeres fue del $15,8 \%$ mientras que para los varones fue sólo del 7,8\%. Esta tendencia ratifica lo anteriormente indicado sobre la feminización de la matricula del nivel superior.

\section{Gráfico № 3 \\ J óvenes entre 25 y 29 años según máximo nivel educativo alcanzado Total del País}

Fuente: Elaboración propia en base a datos de los Censos Naciones de Población y Viviendas del INDEC.

En síntesis, un conjunto de investigaciones han señalado que en nuestro país, sobre todo en las últimas décadas, se produjo un aumento significativo del perfil educativo de la población en general, y de la fuerza de trabajo en particular, con especial relevancia entre los jóvenes (Filmus D., Kaplan C., Miranda A. y Moragues M. 2001; Groisman F. 2003). En este sentido, existe un amplio consenso en que la transformación más sobresaliente fue aquella relativa al incremento de la población que ha obtenido un certificado del nivel medio de enseñanza. En paralelo a estos señalamientos, a partir del análisis realizado puede verificarse como ha sido la distribución del incremento de la escolarización en la población joven. De acuerdo a esta evolución se puede corroborar una expansión del certificado educativo del nivel medio y una tendencia a la igualación entre los años de escolarización entre varones y mujeres, sobre todo en los jóvenes plenos y jóvenes adultos.

Ahora bien, ante un contexto de deterioro laboral, el incremento de las credenciales educativas ha significado una profundización del proceso de devaluación de credenciales (Filmus, 2001) dinámica que, como se ha sostenido en estudios anteriores de este equipo de trabajo (Miranda, Otero y Zelarayan, 2005) se agudiza durante el último período bajo análisis. En el apartado siguiente veremos la evolución de las principales tendencias respecto a la dinámica laboral de los jóvenes.

\section{Tendencias en la inserción laboral de la población joven}

En la Argentina, sobre todo en la década de los noventa, la persistencia de altas tasas de desocupación y la creciente vulnerabilidad de los jóvenes determinaron que la problemática de la desocupación juvenil se convirtiese en un asunto de principal importancia en la agenda pública y fuese objeto de numerosos estudios (Gallart M.A. 1993; Feldman S. 1995; Jacinto C. 1996). En este sentido, hubo consenso entre los especialistas que abordaron la temática en que los jóvenes constituyen uno de los grupos más perjudicados por la crisis del mercado de trabajo. El deterioro que los jóvenes experimentaron en su inserción socio-ocupacional puede implicar amplias consecuencias, de allí la relevancia de la temática.

Siguiendo los datos censales entre el período 1970-2001, entre los jóvenes menores se registró una baja en la tasa de actividad y empleo y un incremento pronunciado en la tasa de desocupación. Una diversidad de estudios demuestraron que al interior de la población joven, el grupo de 15 a 19 años fue el más afectado en términos de desocupación en América Latina (CEPAL, 1999). Asimismo, se ha señalado que para este subgrupo de edad, la pérdida de empleo es paralela a la caída de la actividad económica, dada en forma simultánea a un importante aumento de la escolaridad. A partir del análisis de la información censal, se corroboran estas tendencias.

Como vemos en el cuadro $\mathrm{N}^{\circ} 3$, la tasa de actividad y de empleo fue disminuyendo paulatinamente, mientras que la asistencia escolar mostró un fuerte incremento sobre todo desde mediados de los noventa, incremento que marchó en paralelo a la implementación de la Ley Federal de Educación. Además, podemos mencionar que la tasa de asistencia de este subgrupo etario muestra un aumento del 33\% entre 1970-2001. En el primer período bajo análisis, la tasa de asistencia alcanzaba un 35,5\%, mientras que en el último período asciende al 68,5\%. De este modo, en 1970 sólo tres de cada diez jóvenes de 15 a 19 años asistía a la escuela, mientras que en el 2001 asistían seis de cada diez. Cabe destacar también que en forma paralela, la tendencia al incremento se verifica tanto entre los varones como entre las mujeres.

A partir de los indicadores laborales al interior del subgrupo podemos señalar un conjunto de tendencias interesantes de destacar. La tasa de actividad en 1970 era del 46\%, descendiendo al 31\% hacia principios del siglo XXI. En cuanto a la distribución por género, este descenso de la tasa de actividad se verifica tanto en mujeres como en varones, sin embargo entre estos últimos es entre quienes esta disminución es mayor.

En una misma dirección la tasa de empleo en los jóvenes menores disminuyó del 43,4\% al 11,6\% entre 1970-2001, respectivamente. La caída más pronunciada de la tasa de empleo se observa entre los relevamientos del último período, es decir 1991-2001, la diferencia porcentual es de $-20,4 \%$. Por otra parte, la tasa de desocupación se eleva del 5,6\% al $17,8 \%$ entre 1970 y 1991 , 
respectivamente, alcanzando en el 2001 su pico más alto.

Dentro de este panorama, los jóvenes menores experimentaron una pérdida de empleo de tal magnitud que, actualmente, se dificulta hablar de mercado de trabajo en términos estrictos para este subgrupo de edad. Justamente, durante el último censo sólo 1.1 de cada 10 jóvenes entre 15 y 19 años contaba con una ocupación. Si bien es cierto que, el censo 2001 fue realizado durante la antesala de una de las crisis más graves de nuestro país, la tasa de empleo entre los jóvenes de este subgrupo de edad hoy día no es muy distinta. Más aun, si observamos entre puntas el período analizado, podemos verificar que -sobre todo para los varones- mientras a principios de los años '70 la inserción laboral representaba una opción habilitada para quienes no continuaban en la educación secundaria, actualmente dicha opción parece haber perdido vigencia (Ver cuadro № 2).

Cuadro № 2

Evolución de las tasas de escolaridad, actividad, empleo y desocupación entre los jóvenes de 15 a 19 años - Total del País

\begin{tabular}{|c|c|c|c|c|c|}
\hline & $\mathbf{1 9 7 0}$ & $\mathbf{1 9 8 0}$ & $\mathbf{1 9 9 1}$ & $\mathbf{2 0 0 1}$ & $\begin{array}{c}\text { Diferencia } \\
\mathbf{2 0 0 1 - 1 9 7 0}\end{array}$ \\
\hline Asiste & 35.5 & 42.5 & 54.3 & 68.5 & 33.0 \\
\hline Tasa de Actividad & 46.0 & 39.7 & 38.9 & 31.4 & -14.6 \\
\hline Tasa de Empleo & 43.4 & S/D & 32.0 & 11.6 & -31.8 \\
\hline Tasa de Desocupación & 5.6 & S/D & 17.8 & 63.0 & 57.3 \\
\hline \multicolumn{5}{|c|}{ HOMBRES } \\
\hline Asiste & 36.0 & 40.3 & 51.8 & 66.2 & 30.2 \\
\hline Tasa de Actividad & 60.7 & 51.6 & 48.6 & 36.7 & -24.0 \\
\hline Tasa de Empleo & 57.3 & S/D & 40.8 & 15.5 & -41.8 \\
\hline Tasa de Desocupación & 5.5 & S/D & 15.9 & 21.1 & 15.6 \\
\hline \multicolumn{7}{|c|}{ MUJ ERES } \\
\hline Asiste & 34.9 & 43.0 & 56.7 & 70.8 & 35.8 \\
\hline Tasa de Actividad & 31.0 & 27.7 & 29.6 & 25.9 & -5.1 \\
\hline Tasa de Empleo & 29.2 & S/D & 23.4 & 7.5 & -21.6 \\
\hline Tasa de Desocupación & 5.8 & S/D & 20.9 & 70.7 & 64.8 \\
\hline
\end{tabular}

Fuente: Elaboración propia en base a datos del Censo Nacional de Población y Viviendas del INDEC.

En relación con los jóvenes plenos, las tendencias indican que a diferencia de lo que ocurre entre el subgrupo analizado anteriormente, la tasa de actividad permanece constante a lo largo de todo el período, asumiendo valores mayores al $60 \%$. Entre tanto, la tasa de empleo muestra una disminución pronunciada (un 25\%). En el primer período censal -1970-, la tasa de empleo alcanzaba un 62,8\% y durante el último relevamiento un $38,5 \%$, observándose una variación interperíodo de $-24,2 \%$. Estos datos corroboran que el período se ha caracterizado por la pérdida sostenida de empleo y por el aumento de la tasa de desocupación que asciende a un $27,2 \%$ en el 2001 (2).

Estas transformaciones en la inserción laboral de los jóvenes se verifican tanto en la caída de la actividad del subgrupo como en el respectivo aumento de la asistencia al sistema de educación formal, siendo las mujeres como hemos visto quienes presentan los mayores porcentajes de asistencia.

Tomando en cuenta la evolución de la tasa de actividad, se observa un incremento significativo de la presencia de la mujer en el mercado laboral. En los varones durante 1970 la tasa de actividad era del 86,3\% disminuyendo al 75,5\% en el 2001; mientras que para el caso de las mujeres un $43,6 \%$ en el primer período, ascendiendo al 55,9\% en el último. Tales indicadores muestran que en el último período censal, la diferencia porcentual de la tasa de actividad entre ambos géneros alcanzó el 20\% (3).

Este acercamiento entre ambos géneros también se da respecto a los indicadores de empleo. En este sentido, en el período de inicio de nuestra exploración es decir 1970, el valor que asumía la tasa de empleo en los jóvenes varones alcanzaba el 83,7\% y en las mujeres era del 42,1\%, así la diferencia porcentual entre ambas tasas superaba el 40\%. Sin embargo, para el 2001 el panorama mostraba modificaciones significativas. Si bien en ambos géneros se registró una disminución en la tasa de empleo, entre los varones esta baja es del -35\%, mientras que para las mujeres la variación es del -13\%. De manera tal que, la tasa de empleo asumió un $48,2 \%$ y $28,9 \%$, en varones y mujeres respectivamente. Asimismo, para ambos se registra un incremento de la tasa de desocupación que asciende abruptamente en el último tramo bajo análisis 1991-2001, siendo mayor para el caso de las mujeres (ver Cuadro № 3).

\section{Cuadro № 3}

Evolución de las tasas de escolaridad, actividad, empleo y desocupación entre los jóvenes de 20 a 24 años - Total del País

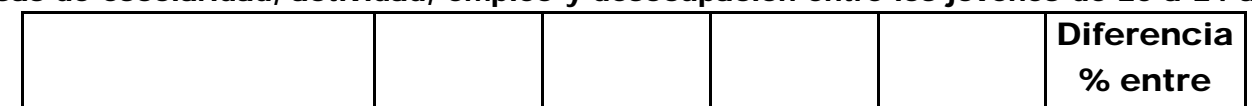




\begin{tabular}{|l|c|c|c|c|c|} 
& $\mathbf{1 9 7 0}$ & $\mathbf{1 9 8 0}$ & $\mathbf{1 9 9 1}$ & $\mathbf{2 0 0 1}$ & $\begin{array}{c}\mathbf{2 0 0 1 -} \\
\mathbf{1 9 7 0}\end{array}$ \\
\hline Asiste & 12.2 & 15.1 & 24.1 & 30.8 & 18.6 \\
\hline Tasa de Actividad & 64.8 & 63.6 & 67.5 & 65.7 & 0.9 \\
\hline Tasa de Empleo & 62.8 & S/D & 60.0 & 38.5 & -24.2 \\
\hline Tasa de Desocupación & 3.1 & S/D & 11.0 & 27.2 & 24.0 \\
\hline \multicolumn{5}{|c|}{ HOMB RES } \\
\hline Asiste & 13.5 & 14.2 & 22.9 & 27.9 & 14.4 \\
\hline Tasa de Actividad & 86.3 & 82.2 & 82.9 & 75.5 & -10.8 \\
\hline Tasa de Empleo & 83.7 & S/D & 75.3 & 48.2 & -35.4 \\
\hline Tasa de Desocupación & 3.0 & S/D & 9.1 & 36.1 & 33.1 \\
\hline \multicolumn{5}{|c|}{ MUJ ERES } \\
\hline Asiste & 10.8 & 13.7 & 25.2 & 33.7 & 22.8 \\
\hline Tasa de Actividad & 43.6 & 42.2 & 52.4 & 55.9 & 12.3 \\
\hline Tasa de Empleo & 42.1 & S/D & 45.1 & 28.9 & -13.2 \\
\hline Tasa de Desocupación & 3.3 & S/D & 13.9 & 48.3 & 44.9 \\
\hline
\end{tabular}

Fuente: Elaboración propia en base a datos del Censo Nacional de Población y Viviendas del INDEC.

Finalmente los indicadores laborales para aquellos que hemos denominado como jóvenes adultos, señalan el aumento sostenido de la participación en el mercado de trabajo ( 9,4 puntos entre los extremos) y un incremento en la tasa de escolarización. Al mismo tiempo se verificó un descenso en el empleo que es menor al registrado en los otros subgrupos etarios analizados. La tasa de actividad, durante el primer relevamiento analizado alcanzó 65,7\%, elevándose 10 puntos porcentuales en el último período censal. La tasa de empleo sufrió una disminución de igual magnitud en lo que va del período. Y respecto a la tasa de desocupación, ascendió al 26,4\% en el 2001, verificando un incremento de casi un 25\% entre 1970-2001 (ver Cuadro № 4).

Si tomamos en cuenta la variable género, entre los varones la tasa de actividad en el período histórico estudiado disminuye un 7,9\%, por el contrario, en las mujeres se incrementa un $26,6 \%$. El aumento en la tasa de actividad de las mujeres y la disminución de dicha tasa en el caso de los varones nos lleva a reflexionar en la tendencia, ya señalada en los jóvenes plenos, sobre la equiparación entre géneros. El aumento en la tasa de actividad de las mujeres es significativo en tanto parece indicar un acercamiento con los valores registrados para el caso de los varones. Si, en 1970 la diferencia entre las tasas de actividad de varones y mujeres era del $60 \%$, en el 2001 esa diferencia es solamente del 25\%.

El acercamiento entre géneros se expresa también en la evolución de la tasa de empleo. En los varones, la tasa disminuyó un -25,9\% entre 1970-2001, y en las mujeres la tasa se incrementó un 6,8\% durante el mismo período. Así en referencia al último censo, puede señalarse que la tasa de actividad alcanzó el $68,4 \%$ y $42,4 \%$ para varones y mujeres respectivamente. $Y$ en cuanto a la tasa de desocupación, en ambos géneros se registraron aumentos, sin embargo entre las mujeres éste ha sido mayor (ver cuadro № 4).

En síntesis, los datos nos indican un incremento en la participación de las mujeres en el mercado laboral, bien intenso entre las jóvenes de 25 a 29 años. Al tiempo que, puede observarse una caída en la participación económica entre los jóvenes varones. Estas tendencias comienzan a ser más notorias a partir de la década de los noventa (ver cuadro $N^{\circ} 4$ ). De manera tal que, pareciera que los comportamientos de varones y mujeres se van homologando.

\section{Cuadro № 4}

Evolución de las tasas de escolaridad, actividad, empleo y desocupación entre los jóvenes de 25 a 29 años - Total del País

\begin{tabular}{|l|c|c|c|c|c|}
\hline & & & & & $\begin{array}{c}\text { Diferencia \% } \\
\text { entre 2001- } \\
\mathbf{1 9 7 0}\end{array}$ \\
\hline Asiste & $\mathbf{1 9 7 0}$ & $\mathbf{1 9 8 0}$ & $\mathbf{1 9 9 1}$ & $\mathbf{2 0 0 1}$ & 8.7 \\
\hline Tasa de Actividad & 4.7 & 6.8 & 9.6 & 13.5 & 9.4 \\
\hline Tasa de Empleo & 65.7 & 65.3 & 73.4 & 75.2 & -9.4 \\
\hline Tasa de Desocupación & 1.5 & S/D & 5.6 & 26.4 & 24.9 \\
\hline \multicolumn{7}{|c|}{ HOMBRES } \\
\hline Asiste & 5.8 & 6.5 & 10.2 & 13.5 & 7.6 \\
\hline Tasa de Actividad & 95.8 & 93.8 & 93.0 & 87.8 & -7.9 \\
\hline Tasa de Empleo & 94.3 & S/D & 88.9 & 68.4 & -25.9 \\
\hline Tasa de Desocupación & 1.4 & S/D & 4.4 & 22.0 & 20.6 \\
\hline \multicolumn{7}{|c|}{ MUJ ERES }
\end{tabular}




\begin{tabular}{|l|c|c|c|c|c|}
\hline Asiste & 3.7 & 5.1 & 9.0 & 15.2 & 11.5 \\
\hline Tasa de Actividad & 36.2 & 37.4 & 54.3 & 62.8 & 26.6 \\
\hline Tasa de Empleo & 35.6 & S/D & 50.2 & 42.4 & 6.8 \\
\hline Tasa de Desocupación & 1.7 & S/D & 7.5 & 32.4 & 30.7 \\
\hline
\end{tabular}

Fuente: Elaboración propia en base a datos del Censo Nacional de Población y Viviendas del INDEC.

A partir de los indicadores laborales y educativos analizados, nos parece importante señalar algunas tendencias que se han advertido en relación con el modelo del núcleo familiar. En este sentido, durante la década del setenta se señalaba la "familia nuclear", como modelo predominante de organización doméstica en los centros urbanos. Este modelo se estructuraba sobre la base de la figura de un adulto hombre proveedor -único sostén de familia-, y una mujer "ama de casa" que ocupaba su tiempo en la educación de las nuevas generaciones y del cuidado del hogar (Carnoy M. 2000).

La consolidación y estandarización de dicho modelo implicó que, la participación laboral de las mujeres fluctuara con el ingreso de las jóvenes en su etapa reproductiva. Los estudios demográficos durante aquella época demostraban que la actividad económica de las mujeres estaba fuertemente correlacionada con su estado civil, ya que era frecuente su inactividad a partir de la llegada del primer hijo entre las mujeres casadas (Lattes Z Recchini de y Lattes A. 1974). La continuidad en las tendencias hacia la menor participación laboral y escolar de las mujeres llevó a que en los estudios se subrayara un fenómeno de "domesticidad excluyente" entre las jóvenes. En un mismo sentido, estudios advertían para aquella época que una gran proporción de mujeres jóvenes no estudiaba, ni trabajaba, y se insertaba socialmente sólo en el ámbito familiar (Braslavsky C. 1986).

No obstante, en el marco de los cambios económicos, sociales y culturales producidos en los últimos años, pareciera que las elecciones de los jóvenes en relación con la constitución de una familia están siendo postergadas (Torrado S., 2003). Numerosas investigaciones han advertido que, hace 20 años, el proyecto de formar una familia se concretaba a más temprana edad. El aletargamiento en la constitución de un núcleo familiar propio, marcha a la par de las tendencias manifiestas en relación con el incremento en la participación de las mujeres en la actividad económica.

A pesar de que tales mutaciones traducen trastrocamientos sociales y culturales profundos del escenario global, también son el producto de situaciones coyunturales. En este sentido, en Argentina durante las últimas décadas, algunos estudios señalaron la influencia directa del desempleo y la precarización del empleo sobre la edad de inicio de la unión conyugal, del comienzo de la etapa de procreación, de quién es la persona que mantiene económicamente a la familia (jefe de hogar) y qué ocupación tiene esta persona (4).

\section{Comentarios finales}

A principios del siglo XXI la población joven representa casi un cuarto de la población total del país. En el primer apartado del presente artículo hemos podido observar que desde la década de 1970 a la actualidad el peso específico de este sector de la población se ha mantenido relativamente constante. Si bien no se han registrado importantes variaciones en la posición que ocupan dentro de la pirámide poblacional, el contexto ha cambiado significativamente para las actuales generaciones de jóvenes.

Las transformaciones sociales y económicas de los últimos treinta años fueron modificando las condiciones estructurales en las cuales transcurre la inserción laboral de los jóvenes. Ante este panorama, a partir de la lectura de los datos bajo análisis, hemos visto cómo históricamente se observan un conjunto de tendencias destacadas en torno a indicadores laborales y educativos. Dentro de este conjunto subrayamos dos procesos centrales que atraviesan a la población joven de nuestro país: 1) una tendencia hacia la mayor escolarización y 2) un incremento en la tasa de desocupación.

Respecto al primer proceso, a lo largo de los períodos intercensales puede corroborarse una tendencia en cuanto al incremento del nivel educativo de los jóvenes, registrándose las mayores variaciones en el nivel de enseñanza secundario. En este marco entre los jóvenes menores, el incremento del nivel medio alcanzó cerca del 5\%, mientras que entre los jóvenes plenos fue mayor, superando el 25\%. También entre los jóvenes adultos, se registró un fuerte aumentó en el nivel educativo medio (21,4\%). Fue además en este subgrupo donde el incremento del nivel superior de educación ha sido mayor (9 punto porcentuales entre 1970-2001). Pese a ello, como hemos visto estos logros educativos no abarcan al total de la población de jóvenes en Argentina. Aún en el siglo XXI sigue existiendo un número importante de jóvenes que no obtienen el título secundario.

Por otra parte, en referencia a los indicadores educativos es destacable un incremento en la participación de las jóvenes mujeres en el sistema educativo formal. Es decir que desde 1970 hasta la actualidad, el incremento de la escolarización de la población estuvo acompañado por la obtención de mayores certificaciones para las mujeres.

No obstante, a pesar de la evolución creciente de los niveles educativos para los jóvenes en Argentina, existen distintas posibilidades de acceso a una educación de calidad, las mismas se encuentran estrechamente vinculadas con el sector social al que pertenecen. Como sostienen gran parte de las investigaciones abocadas a la temática, aunque se ha masificado el acceso a la educación del nivel primario y tendencialmente se verifica un aumento de la asistencia en el nivel secundario, esta mayor inclusión de jóvenes en el sistema educativo formal se da en el marco de la persistencia del deterioro en su calidad (Jacinto, C. 2004). Es decir, este proceso de 
inclusión no garantizó el acceso a una educación de calidad para el conjunto de la población de jóvenes. En este sentido, hay consenso en que uno de los principales desafíos de la educación argentina hoy, gira en torno al mejoramiento de una oferta de calidad del bien educativo.

Los debates actuales con relación al sistema educativo involucran no sólo cuestiones de acceso, sino que también abarcan replanteos sobre el rol social de la escuela en nuestras sociedades. En particular en nuestro país, durante los noventa se ha enfatizado que es el nivel de educación media aquel que atraviesa una de las crisis más profundas. Y en este marco adquiere centralidad la pregunta por la función de la escuela secundaria en el contexto actual (Filmus D, 2001).

En lo que se refiere a los indicadores laborales la problemática de la desocupación ha ido en incremento desde 1970 al 2001 , pero fue durante el último período inter-censal donde adquiere significativa relevancia (5). La desocupación ha afectado al conjunto de la población joven con distintos niveles de intensidad entre los subgrupos etarios. En este sentido, la tasa de desocupación de los jóvenes menores es mayor que la de los jóvenes plenos y adultos. A lo largo del período tanto la tasa de actividad como la de empleo fueron disminuyendo en forma paulatina. No obstante, la escasa relevancia cuantitativa del empleo en el subgrupo de los jóvenes menores se da en paralelo a la evolución de su escolaridad. Para los jóvenes plenos y adultos la tasa de desocupación se incrementa significativamente durante el último período censal, tanto para los varones como para las mujeres aunque es entre éstas donde asumen los mayores porcentajes.

Los indicadores laborales parecen expresar una tendencia hacia la equiparación en la participación de varones y mujeres en el mercado de trabajo. El aumento de la participación femenina en el mercado de trabajo y su profundización en los años noventa ha sido señalado en anteriores investigaciones. Al respecto, Rosalía Cortés advierte que durante el período 1994-2002 aumentan las tasas de participación y de empleo femeninas, en paralelo al incremento de la tasa de desocupación. Situación que está vinculada a diversos procesos, culturales y sociales, de largo plazo que incentivaron la participación femenina, y también la terciarización del producto y de la demanda de trabajo, que se reorientó hacia la oferta femenina. La participación femenina en la fuerza de trabajo creció durante los noventa, en parte impulsada por la caída del empleo de los jefes de hogar y de los ingresos familiares, aunque la creación de empleo no fue suficiente para absorber este aumento (6).

Las tendencias señaladas en los indicadores laborales y educativos de largo plazo en la población joven, se vinculan con cambios en la estructura social y económica operados en nuestras sociedades. Si bien aquí hemos señalamos solo algunas, estas tendencias se dan en el marco de procesos culturales de largo alcance que afectan en los comportamientos de la población joven. Ahora bien, estos cambios ¿qué impacto generan en las transiciones de los jóvenes hacia la vida adulta?, ¿pueden estar limitando el proceso de autonomización, es decir el logro de la emancipación del núcleo familiar de origen?

En este sentido, aún sin poder elaborar respuestas acabadas, consideramos que las restricciones en el acceso a un empleo constituyen una problemática central que afecta sus posibilidades de autonomización. El logro de una plena independencia económica o de la conformación de un hogar propio sugiere en la actualidad para mucho de nuestros jóvenes un camino de amplias dificultades.

\section{ANEXO}

\section{Cuadro № 1}

Evolución de la población de jóvenes entre 15 y 29 años - valores absolutosTotal del País

\begin{tabular}{|c|c|c|c|c|c|}
\hline & 1970 & 1980 & 1991 & 2001 & 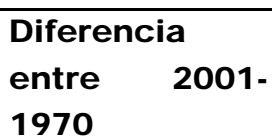 \\
\hline 15 a 19 años & 2098700 & \begin{tabular}{|l|}
2341488 \\
\end{tabular} & 2850105 & 3188304 & \begin{tabular}{|l|}
1089604 \\
\end{tabular} \\
\hline 20 a 24 años & 1950500 & 2224157 & 2454123 & 3199339 & 1248839 \\
\hline 25 a 29 años & 1702700 & 2124283 & 2304242 & 2695341 & 992641 \\
\hline 15 a 29 años & 5751900 & 11255573 & 7608470 & 9049984 & 3298084 \\
\hline \multicolumn{6}{|c|}{ HOMBRES } \\
\hline 15 a 19 años & 1058850 & 1173841 & 1417619 & 1613030 & 554180 \\
\hline 20 a 24 años & 969950 & 1099810 & 1213835 & 1597939 & 627989 \\
\hline 25 a 29 años & 842550 & 1050065 & 1137361 & 1329493 & 486943 \\
\hline 15 a 29 años & 2871350 & 3323716 & 3768815 & 4540462 & 1669112 \\
\hline \multicolumn{6}{|c|}{ MUJ ERES } \\
\hline 15 a 19 años & 1039850 & 1167647 & 1432486 & 1575274 & 535424 \\
\hline 20 a 24 años & 980550 & 1124347 & 1240288 & 1601400 & 620850 \\
\hline 25 a 29 años & 860150 & 1074218 & 1166881 & 1365848 & 505698 \\
\hline 15 a 29 años & 2880550 & 3366212 & 3839655 & 4542522 & 1661972 \\
\hline
\end{tabular}


Gráfico № 1

J óvenes entre 15 y 19 años según máximo nivel educativo alcanzado y género

Total del País

Fuente: Elaboración propia en base a datos del Censo Nacional de Población y Viviendas del INDEC

Gráfico № 2

J óvenes entre 20 y 24 años según máximo nivel educativo alcanzado y género

Total del País

Fuente: Elaboración propia en base a datos del Censo Nacional de Población y Viviendas del INDEC

Gráfico № 3

J óvenes entre 25 y 29 años según máximo nivel educativo alcanzado y género

Total del País

Fuente: Elaboración propia en base a datos del Censo Nacional de Población y Viviendas del INDEC

\title{
Gráfico № 4 \\ Población de 30 años y más según máximo nivel educativo alcanzado Total del País
}

Fuente: Elaboración propia en base a datos de los Censos Naciones de Población y Viviendas del INDEC

\author{
Gráfico № 5 \\ Población de 30 años y más según máximo nivel educativo alcanzado y género \\ Total del País
}

Fuente: Elaboración propia en base a datos de los Censos Naciones de Población y Viviendas del INDEC

\section{Notas}

(1) A lo largo del texto usaremos indistintamente el término nivel medio y escuela secundaria.

(2) En anteriores investigaciones realizadas por este equipo de trabajo, señalábamos que la mayor desocupación entre los jóvenes es un fenómeno observado desde fines de los años sesenta que fue interpretado como "desempleo de inserción", es decir, asociado a las dificultades en la obtención del primer empleo (Llach J.J, 1978). A mediados de los ochenta, para el grupo de jóvenes de 20 a 24 años la tasa de desocupación asumía un valor 2.3 veces mayor que el correspondiente al de 30 años y más.

(3) En 1970, la diferencia porcentual de la tasa de actividad entre varones y mujeres era del $42,7 \%$.

(4) En este sentido, Susana Torrado en un reciente trabajo publicado en el 2005 señala que: “(...) con posterioridad a 1990, cuando empieza a deteriorarse la situación del mercado de trabajo, se constata lo siguiente: el número anual de matrimonio y nacimientos por 1.000 habitantes aceleró bruscamente su caída; la progresión del porcentaje de los extramatrimoniales en el total de nacimientos también se hizo más rápido; el peso tendencialmente ascendente de las uniones consensuales también se aceleró. Y tales hechos se verifican en todo el país. A pesar de estas menciones, la autora advierte que falta mayor información estadística para aproximarse a los cambios que están sucediendo respecto ha la organización de la familia en nuestro país. Cita del libro "Trayectorias nupciales, familias ocultas", Susana Torrado (directora), editorial Miño y Dávila, Buenos Aires, 2005, Pág. 39.

(5) Este fenómeno no es nuevo y ya en la década del ochenta, investigadores argentinos advertían que el problema del desempleo juvenil podría llegar a transformarse en crónico. $Y$ en este caso subrayaban que si no se presta especial atención a este problema la Argentina habría dividido su juventud aun más profundamente. La línea divisoria pasaría entre la generación de la modernización, por un lado, y la generación de la desocupación, por el otro. Las consecuencias de una situación de este tipo para la estabilización democrática serían imprevisibles", Braslavsky C., "La juventud Argentina: entre la herencia del pasado y la construcción del futuro", Revista CEPAL № 29, Santiago de Chile, agosto de 1986, pág. 52-54.

(6) Rosalía Cortés, "Mercado de trabajo y género. El caso Argentino, 1994-2002", en Mujeres, pobreza y mercado de trabajo. Argentina y Paraguay, pág. 69-71.

\section{Bibliografía}

BRASLAVSKY C., "La juventud Argentina: entre la herencia del pasado y la construcción del futuro", Revista CEPAL No 29, Santiago de Chile, agosto de 1986.

CARCIOFI, R. (1983). Educación y aparato productivo en la Argentina, 1976-1982. Un balance de los estudios existentes. El Proyecto Educativo Autoritario. B. y. C. Tedesco. Buenos Aires, FLACSO. 
CARNOY M., "El trabajo flexible en la era de la información", Capítulos 1 y 2, Alianza ensayo, Buenos Aires, 2001. CEPAL-OIJ (2004). La Juventud en Iberoamérica: tendencias y urgencias. Santiago de Chile.

JACINTO Claudia, coordinadora, "Educar para que trabajo?: Discutiendo rumbos en América Latina", Buenos Aires, RedEtis, IIPEIDES/MECyT/ MTEySS, La Crujía, 2004.

CORTES, R. (2003). Mercado de trabajo y género, el caso argentino, 1994-2002. Mujeres, Pobreza y Mercado de Trabajo: Argentina y Paraguay. M. E. Valenzuela. Santiago de Chile, OIT.

DINIECE (2003). Tendencias recientes en la escolarización y la terminalidad del nivel medio de enseñanza. Buenos Aires, Ministerio de Educación, Ciencia y Tecnología de la Nación.

FELDMAN S. (1995). El trabajo de los adolescentes. Construyendo futuro o consolidando la postergación social. Ponencia UNICEF CID CENEP, Buenos Aires.

FILMUS D, C. K., A. MIRANDA Y M. MORAGUES (2001). Cada vez más necesaria, cada vez más insuficiente: la escuela media y mercado de trabajo en épocas de globalización. Buenos Aires, Santillana.

GALLART M.A., M. M. y. M. C. (1993). Educación y empleo en el Gran Buenos Aires 1980-1991. Situación y perspectivas de investigación. Buenos Aires, CENEP.

GROISMAN, F. (2003). "Devaluación educativa y segmentación en el mercado de trabajo del área metropolitana de Buenos Aires entre 1974 y 2000". Estudios del Trabajo № 25.

JACINTO, C. (1996). "Desempleo y transición educación-trabajo en jóvenes de bajos niveles educativos. De la problemática actual a la construcción de trayectorias". Revista Dialógica V.1.

INDEC (Instituto de Estadística y Censos), Censo Nacional de Población, Familias y Viviendas 1970.

INDEC (Instituto de Estadística y Censos), Censo Nacional de Población y Vivienda 1980, Serie D, Total del País, Buenos Aires, 1984.

INDEC (Instituto de Estadística y Censos), Censo Nacional de Población y Vivienda 1991, Serie B, No 25, Total del País, INDEC, Buenos Aires, 1992.

INDEC (Instituto de Estdística y Censos, Censo Nacional de Población, Hogares y Vivienda 2001, Página Web http://www.indec.gov.ar OIT (2004). Tendencias mundiales en el empleo juvenil. Ginebra, Oficina Internacional del Trabajo.

MIRANDA Ana, OTERO Analía y ZELARAYAN Julio, "Distribución de la educación y desigualdad en el empleo: los jóvenes en la Argentina contemporánea", aset $7^{\circ}$ Congreso Nacional de Estudios del Trabajo, del 10 al 12 de agosto de 2005, Buenos Aires.

SALVIA Agustín y TUÑON Ianina, "La situación juvenil en la Argentina durante la última década. Un balance al fin de la convertibilidad", Serie Temas, en Los jóvenes trabajadores frente a la educación, el desempleo y el deterioro social en la Argentina, Fundación Ebert Stiftung-Argentina, 2003.

TORRADO Susana (directora), "Trayectorias nupciales, familias ocultas", editorial Miño y Dávila, Buenos Aires, 2005.

TORRADO Susana, "Historia de la familia moderna (1870-2000)", Ediciones de la flor, Buenos Aires, 2003. 